\title{
An Effective Heuristic Algorithm for Robust Supply Chain Network Design under Uncertainty
}

\author{
Maozhu Jin*, Ruimin Ma, Lifei Yao, Peiyu Ren \\ Buisness School, Sichuan University, Chengdu 610064, China
}

Received: 15 Apr. 2013, Revised: 16 Aug. 2013, Accepted: 18 Aug. 2013

Published online: 1 Mar. 2014

\begin{abstract}
This paper addressing a study on robust supply chain network design under uncertainty environment. We use decomposition and coordination strategy to decompose the model as two parties: the first part is facility location decision which only concludes $0-1$ decision variable, tabu search algorithm is used to determine the $0-1$ decision variables, and then regard the $0-1$ variables as known input parameters. The second part is flow decision, all-or-nothing method is proposed to design the capacity of the facility. From the numerical example, we can see that, the model and algorithm is valid and effective, the robust optimization model not only reduces the risk of market, but avoids the error from the shortage cost.
\end{abstract}

Keywords: Supply Chain Network ; Uncertainty; Robust optimization; Heuristic algorithm

\section{Introduction}

Supply chain networks(SC networks) have emerged as the backbones of economic activities in the modern world. Their importance to the timely and efficient delivery of products as varied as food, energy, clothing, computer hardware, and even toys, etc., has fueled an immense interest in their analysis on the part of both researchers and practitioners. A fundamental topic underlying SC networks, their formulation, analysis, and solution, is that of SC networks design, which may be viewed as foundational to SC management. According to [1], SC network design is the most basic decision of SC management, which influences all other decisions concerning an SC and has the most extensive effect on the chains return on investment and its overall performance. Lin [2] define SC networks design as an integrated configuration of supply, manufacturing and demand side sub-systems. SC networks design involves strategic decisions on the number, location, capacity and mission of the production distribution facilities of a company, or of a set of collaborating companies, in order to provide goods to a predetermined, but possibly evolving, customer base [3].

Many deterministic supply chain networks design models are available in the literature $[4,5,6,7]$. But, in practice, in recent years there have been reported many events that have led to disturbances in supply chains processes (e.g. supplier failures caused by natural disasters or fires in the warehouses, delivery delays due to traffic accidents, product recalls due to lack of fulfilment of quality or safety requirements, etc.). Because of that, there is increasing interest by practitioners and academics to reduce supply chain vulnerability and design robust supply chains.

Robust optimization, presented by Mulvey [8], is able to tackle the decision makers' favored risk aversion or service-level function, and has yielded a series of solutions that are progressively less sensitive to realizations of the data in a scenario set. Then $\mathrm{Yu}$ and $\mathrm{Li}$ [9] improved the robust optimization formulation.

Santoso [10] considered a three-tiered multi-product SC network comprising of supplier, processing facilities and customers. Their model determined the configuration of an SC which consisted of deciding which of the processing centres to build (major configuration decisions) and which processing and finishing machines to procure (minor configuration decisions). Azaron [11] developed a multi-objective stochastic programming approach for SC design under uncertainty. Demands of markets, supplies of suppliers, processing, transportation and capacity expansion costs were all considered as uncertain parameters. Schütz [12] formulated the

\footnotetext{
*Corresponding author e-mail: jinmaozhu@scu.edu.cn
} 
multi-commodity SC design problem as a two-stage stochastic model. The first decision stage of this two-stage model consisted of strategic location decisions, whereas the second stage consists of operational decisions. Feng and Rakesh [13] considered an integrated optimization of logistics and production costs associated with the supply chain members based on the scenario approach to handle the uncertainty of demand. The formulation was a robust optimization model with expected total costs, cost variability due to demand uncertainty, and expected penalty. BenTa et al. [14] proposed a methodology to generate a robust logistics plan that can mitigate demand uncertainty in humanitarian relief supply chains. Pishvaee [15] proposed a robust optimization model for handling the inherent uncertainty of input data in a closed-loop supply chain network design problem. Hahn [16] used Robust optimization methods to deal with operational risks in physical and financial supply chain management due to the uncertainty of future events. Atefeh Baghalian [17] had developed a stochastic mathematical formulation for designing a network of multi-product supply chains comprising several capacitated production facilities, distribution centres and retailers in markets under uncertainty.

But the above literature all assumed the facilities' capacity to be known, we consider that the capacity is determined according the supply chain network and it is unknown.

In this paper, we present a robust optimization model to design SC networks under uncertainty, and a effective heuristic algorithm is proposed for the large-scale problem, tabu-search method is used to solve the problem. The SC networks design mainly includes three decision making: the site of the facility, the capacity of the facility, and the flow between the facility.

\section{Model Formulation}

In this paper, we build the model from the point of the cost and the robustness. Through adjusting the weight of the two objective function to make a balance between them. The indices, parameters and variables used to formulate the concerned supply chain network design problem are described below.

$S$ suppliers, $M$ manufacturers, $I$ distribution centers, $J$ customs, $R$ raw materials, $P$ product types, $\xi$ scenarios

$x_{s m}=1$, if raw materials of the manufacturer $m$ are provide by supplier $s$; otherwise $x_{s m}=0 x_{r s}$, if supplier $s$ provide raw material $r$

$Z_{i}=1$, if a distribution center $i$ is opened; otherwise $Z_{i}=1$

$Y_{m}=1$, if a manufacturer $m$ is opened; $Y_{m}=0$

$z_{i j}=1$, if products of custom $j$ are provided by distribution center $i$; otherwise $z_{i j}=0$ $\hat{q}_{r s m}^{\xi}$ quantity of materials $r$ from supplier $s$ to manufacturer $m$

$\tilde{q}_{p m i}^{\xi}$ quantity of product $p$ from manufacturer $m$ to distribution center $i$

$\bar{q}_{p i j}^{\xi}$ order quantity of product $p$ from distribution center $i$ to custom $j$

$\hat{V}_{m}$ production capacity of manufacture $m$ (hour)

$\tilde{V}_{i}$ capacity of distribution center $i$

\subsection{Model of selecting suppliers}

$\alpha_{r s m}^{\xi}$ unit transportation cost of raw material $r$ from supplier $s$ to manufacture $m$

$\gamma_{r S}^{\xi}$ unit cost of raw material $r$ provided by supplier $s$

$V_{r s m}$ capacity of supplier $s$ providing raw material $r$ for manufacturer $m$

$B_{p r}$ number of units of raw material $m$ required for each unit of product $p$

so the purchase cost can be:

$f_{1}^{\xi}=\sum_{s} \sum_{r} \gamma_{r s}^{\xi} \sum_{m} \hat{q}_{r s m}^{\xi}+\sum_{s} \sum_{m} \sum_{r} \alpha_{r s m}^{\xi} \hat{q}_{r s m}^{\xi}$

The supply capacity of supplier $s$ for raw material $m$ is either enough or 0. so there are some constraints among $x_{s m}, V_{r s m}$ and $\hat{q}_{r s m}^{\xi}$, that can be express as follows:

$$
\begin{gathered}
\text { if } V_{r s m}=0, x_{s m}=0 \\
\sum_{m} \hat{q}_{r s m}^{\xi} \leq V_{r s m} x_{s m}
\end{gathered}
$$

It is need to according to quantity of product to purchase the raw material $r$, and the quantity of product is the sum of order quantity from all the distribution center.

$$
\sum_{m} \hat{q}_{r s m}^{\xi} \geq \sum_{i} \sum_{p} B_{p r} \tilde{q}_{p m i}^{\xi}
$$

The model of selecting supplier is to minimize the objective function under the constraints.

\subsection{Model of facility decision}

$\tilde{c}_{m}^{\xi}$ fix cost of opening manufacture $m$

$\bar{c}_{i}^{\xi}$ fix cost of opening distribution center $i$

$\hat{\gamma}_{p m}^{\xi}$ cost of producing unit product $p$ by manufacture $m$

$\tilde{\gamma}_{p i}^{\xi}$ cost of processing unit product $p$ by distribution center $i$

$\hat{\alpha}_{p m i}^{\xi}$ unit transportation cost of product $p$ from manufacture $m$ to distribution center $i$

$\tilde{\alpha}_{p i j}^{\xi}$ unit transportation cost of product $p$ from distribution center $i$ to custom $j$ 
$\sigma_{m}^{\xi}$ cost of increasing unit production capacity for manufacturer $m$

$\hat{\sigma}_{i}^{\xi}$ cost of increasing unit processing capacity for distribution center $i$

$\varpi_{p m}$ time of unit product $p$ for manufacturer $m$ (hour)

$D_{p j}^{\xi}$ the demand of product $p$ at custom $j$

The cost of the facility decision is composed of four parts, include fixed $\operatorname{costs}(F C)$, processing $\operatorname{cost}(P C)$, transportation costs $(T C)$, facility capacity purchase cost (FCC).

So the total cost of the facility decision is $f_{2}^{\xi}=F C+$ $P C+T C+F C C$.

$$
\begin{aligned}
& F C^{\xi}=\sum_{m} \tilde{c}_{m}^{\xi} Y_{m}+\sum_{i} \bar{c}_{i}^{\xi} Z_{i} \\
& P C^{\xi}=\sum_{p} \sum_{m} \hat{\gamma}_{p m}^{\xi} \tilde{q}_{p m i}^{\xi}+\sum_{p} \sum_{i} \tilde{\gamma}_{p i}^{\xi} \bar{q}_{p i j}^{\xi} \\
& T C^{\xi} \\
& \sum_{r} \sum_{s} \sum_{m} \hat{q}_{r s m}^{\xi} \alpha_{r s m}^{\xi}+\sum_{p} \sum_{m} \sum_{i} \tilde{q}_{p m i}^{\xi} \hat{\alpha}_{p m i}^{\xi}+\sum_{p} \sum_{i} \sum_{j} \bar{q}_{p i j}^{\xi} \tilde{\alpha}_{p i j}^{\xi} \\
& F C C^{\xi}=\sum_{m} \sigma_{m}^{\xi} \hat{V}_{m}+\sum_{i} \hat{\sigma}_{i}^{\xi} \tilde{V}_{i}
\end{aligned}
$$

And at the same time, the model need satisfy the constraints below:

For distribution center $i$, quantity of product $p$ to customer $j$ should be less than the order quantity from the manufacturer $m$.

$$
\sum_{m} \tilde{q}_{p m i}^{\xi} \geq \sum_{j} \bar{q}_{p i j}^{\xi}
$$

One customer can only purchase product from one distribution center.

$$
\sum_{i} z_{i j}=1
$$

The quantity of the product output is equal with the quantity to the distribution center, and the total output can not be over than the production capacity of the manufacturer.

$$
\sum_{i} \sum_{p} \varpi_{p m} \tilde{q}_{p m i}^{\xi} \leq \hat{V}_{m}
$$

The sum of the quantity of product send into and out from the distribution center can not be over the capacity of the distribution center.

$$
\sum_{m} \sum_{p} \tilde{q}_{p m i}^{\xi}+\sum_{j} \sum_{p} \bar{q}_{p i j}^{\xi} \leq \tilde{V}_{i}
$$

Variable constraints. The quantity of product send from manufacturer to distribution center and distribution center to customer, the capacity of manufacturer and capacity of distribution center can not be negative number, decision variable is $0-1$ integer variable.

$$
\begin{gathered}
\tilde{q}_{p m i}^{\xi} \geq 0, \bar{q}_{p i j}^{\xi} \geq 0 \\
\hat{V}_{m} \geq 0, \tilde{V}_{i} \geq 0
\end{gathered}
$$

$$
Z_{i}, Y_{m}, z_{i j} \in 0,1
$$

\subsection{Robust optimization model of integrate supply chain network design}

Through the analysis of the Model of selecting suppliers and Model of facility decision, we can get the optimization model of supply chain network design through integrate the two model, the new objective function is:

$$
f^{\xi}=f_{1}^{\xi}+f_{2}^{\xi}
$$

For multiple scenario parameters, the value of the objective function become random variable which occur at a certain probability $\rho_{\xi}$, the objective function is not a single value any more. So we can use stochastic programming to express the objective function as average value, that is,

$$
f=\sum_{\xi} \rho_{\xi} f^{\xi}
$$

In order to enhance the robustness of the model, a higher order term which is a feasible penalty function can be added to the objective function.

The variance of the supply and the real demand is

$$
\sigma^{2}=\sum_{\xi} \sum_{i} \sum_{j} \sum_{p} \rho_{\xi}\left(\bar{q}_{p i j}^{\xi}-D_{p j}^{\xi}\right)^{2}
$$

So the penalty function is

$$
R_{1}=\omega \sum_{\xi} \sum_{i} \sum_{j} \sum_{p} \rho_{\xi}\left(\bar{q}_{p i j}^{\xi}-D_{p j}^{\xi}\right)^{2}
$$

$\omega$ is a weight of the objective programming, it can control the weight of the higher order term $\sigma^{2}$ in the objective function. We can get a series of robustness solution through taking different $\omega$ value, thus measure the trade-off between the robustness and the cost.

The model can be improved as follow:

$$
\min f=\sum_{\xi} \rho_{\xi} f^{\xi}+\omega \sum_{\xi} \sum_{i} \sum_{j} \sum_{p} \rho_{\xi}\left(\bar{q}_{p i j}^{\xi}-D_{p j}^{\xi}\right)^{2}
$$

But it neglect the risk which making decision under uncertainty and the difference between the single objective function and the average objective function. So a new penalty term should be added, which is a risk penalty term for the objective function.

$$
R_{2}=\lambda \sum_{\xi} \rho_{\xi}\left(f^{\xi}-\sum_{\xi} \rho_{\xi} f^{\xi}\right)^{2}
$$

$\lambda$ denotes the risk factor of the objective function.

The robust optimization model is

$$
\begin{aligned}
& \quad \operatorname{Minf}=\sum_{\xi} \rho_{\xi} f^{\xi}+\omega \sum_{\xi} \sum_{i} \sum_{j} \sum_{p} \rho_{\xi}\left(\bar{q}_{p i j}^{\xi}-D_{p j}^{\xi}\right)^{2}+ \\
& \lambda \sum_{\xi} \rho_{\xi}\left(f^{\xi}-\sum_{\xi} \rho_{\xi} f^{\xi}\right)^{2} \\
& \quad \text { subject to constraints (1)-(10). }
\end{aligned}
$$




\section{Heuristic}

In order to find the near optimal solution, we use decomposition and coordination strategy to address the model. The supply chain model can be divided into two parts, that are facility location decision and flow decision. The facility location decision includes choosing suppliers, constructing factories and distribution centers, this part just include $0-1$ integer variables. The flow decision means the flow between the nodes, includes quantities of raw material, shipment and so on.

\subsection{Facility location decision}

In this section, we use tabu search algorithm to decide the facility location, the basic structure is as follows:

Step 1. Construction of an initial solution. The solution can be expressed as follow:

$$
\begin{aligned}
& X=(x, Y, Z, z) \\
& \left(\begin{array}{cccc}
1 & 0 & \cdots & 1 \\
0 & 1 & \cdots & 1 \\
\vdots & \vdots & \vdots & \vdots \\
1 & 1 & \cdots & 0
\end{array}\right)_{1 \times R}\left(\begin{array}{c}
1 \\
0 \\
\vdots \\
1
\end{array}\right)_{M \times 1}\left(\begin{array}{c}
0 \\
1 \\
\vdots \\
1
\end{array}\right)_{I \times 1}\left(\begin{array}{cccc}
0 & 0 & \cdots & 0 \\
1 & 0 & \cdots & 1 \\
\vdots & \vdots & \vdots & \vdots \\
0 & 1 & \cdots & 1
\end{array}\right)_{I \times J}
\end{aligned}
$$

Step 2. Construction of neighborhood. Choose two row from matrix $x$, swap the element on the same column, then test feasibility of the new solution. Choose one element whose value is 1 from matrix $Y$ at random, make mutation for the other two points. The processing method of $Z, z$ is as same with $Y, x$.

Step 3. Fitness function. We take the objective function of robust supply chain model as the fitness function.

Step 4. Candidate set. Capturing $\mathrm{N}$ neighbour at random from the adjacent domain.

Step 5. Tabu Length. Set the Tabu Length as $\sqrt{N}$.

Step 6. Aspiration criterion. If all the feasible solution are taboo, then releasing the optimal feasible solution.

Step 7. Stopping criterions. Set the maximum iterative step number $J \times R$ as the stopping criterion.

\subsection{Flow decision}

Minimum path assignment method is also called all-or-nothing assignment method, it is a kind of assignment method that the flow can be accumulated. In this paper, we firstly calculate the unit flow cost between all the nodes, then put all the products equivalent to nodes with minimum unit flow cost and get the capacity of the nodes. The detail procedure is as follows:

Step 1. For anyone distribution center $i\left(Z_{i}=1\right)$ and its upstream node manufacturer $m\left(Y_{m}=1\right)$, calculate the unit flow cost of product $p$ between the nodes. The cost
Table 2 Product consumption

\begin{tabular}{ccccc}
\hline \multirow{2}{*}{ Product $i$} & \multicolumn{4}{c}{ Raw material $r$} \\
\cline { 2 - 5 } & 1 & 2 & 3 & 4 \\
\hline 1 & 2 & 3 & 0 & 0 \\
2 & 2 & 3 & 1 & 0 \\
3 & 2 & 3 & 1 & 2 \\
\hline
\end{tabular}

include the economical cost and environmental impact, under scenario $\xi$ the unit economical cost $\beta_{p m i}^{\xi}=\hat{\gamma}_{p m}^{\xi}+\hat{\alpha}_{p m i}^{\xi}+\sigma_{m}^{\xi}$. Then, we think the products $p$ required by distribution center $i$ are all from manufacturer $m$ with minimum unit flow cost, $m^{*}=m_{p m i}^{\min }$, and get $\tilde{q}_{p m i}^{\xi}=\sum_{j} \bar{q}_{p i j}^{\xi}$.

$$
\text { If } \sum_{i} \sum_{p} \tilde{q}_{p m i}^{\xi}=0, Y_{m}=0 \text {; else } Y_{m}=1 \text {. }
$$

Step 2. As the same with step 1, for any manufacturer $m\left(Y_{m}=1\right)$, put all the demands of raw material equivalent to suppliers with minimum unit flow cost, and modify the value of $x_{s m}$.

Step 3. Getting the capacity of the nodes. The production capacity of manufacturer $m$ is $\hat{V}_{m}=\max _{\xi}\left\{\sum_{i} \sum_{p} \tilde{q}_{p m i}^{\xi}\right\}$, the capacity of distribution center $i$ is $\tilde{V}_{i}=\max _{\xi}\left\{\sum_{i} \sum_{p} \tilde{q}_{p m i}^{\xi}+\sum_{j} \sum_{p} D_{p j}^{\xi} z_{i j}\right\}$.

\section{Numerical example}

In this section, a simplified numerical study is conducted to illustrate the applicability of the proposed method.

\subsection{Detail data}

Set the weight coefficient $\omega$ of objective programming as 0.5 , the risk coefficient as 0.6 , the maximum iterative step length as $J R=1000$, and the tabu length $l=12$.

Uncertain parameters include uncertain demand and uncertain cost, through cross combination of the uncertain parameters for demand and uncertainty cost, and judging its rationality formed a variety of scenario parameters. In this case, there are separately 3 scenarios market demand and uncertain cost, so it formed 9 scenario parameters, the probability of every scenario is $\frac{1}{9}$.

\subsection{Algorithm performance test}

According to the algorithm, we can get the convergence curve of the algorithm as shown in figure 1:

From the figure 1, we can see that, the algorithm become stable after 600 steps, so the tabu-search based on all-or-nothing assignment method is acceptable in the aspect of convergence. 
Table 1 Supply chain network

\begin{tabular}{c|c|c|c|c|c}
\hline Supplier & Manufacturer & Distribution center & Custom & Material & Product \\
\hline 5 & 2 & 5 & 8 & 4 & 3 \\
\hline
\end{tabular}

Table 3 Market demands for Scenario 1

\begin{tabular}{ccccccccc}
\hline \multirow{2}{*}{ Product $i$} & \multicolumn{8}{c}{ Customer $c$} \\
\cline { 2 - 9 } & 1 & 2 & 3 & 4 & 5 & 6 & 7 & 8 \\
\hline 1 & 200 & 250 & 300 & 350 & 200 & 200 & 200 & 350 \\
2 & 150 & 200 & 250 & 300 & 100 & 100 & 150 & 200 \\
3 & 250 & 100 & 300 & 250 & 200 & 100 & 200 & 300 \\
\hline
\end{tabular}

For scenarios 2 and 3 , the estimations are multiplied by 1.1 and 1.2 respectively.

Table 4 Transportation cost connecting with manufacturer $m$ for scenario 1

\begin{tabular}{|c|c|c|c|c|c|c|c|c|c|c|}
\hline \multirow{2}{*}{ Manufacturer $m$} & \multicolumn{5}{|c|}{ Supplier $s$} & \multicolumn{5}{|c|}{ Distribution center $i$} \\
\hline & 1 & 2 & 3 & 4 & 5 & 1 & 2 & 3 & 4 & 5 \\
\hline 1 & 0.08 & 0.06 & 0.03 & 0.04 & 0.02 & 0.08 & 0.08 & 0.09 & 0.01 & 0.08 \\
\hline 2 & 0.04 & 0.02 & 0.06 & 0.05 & 0.08 & 0.06 & 0.07 & 0.05 & 0.05 & 0.1 \\
\hline
\end{tabular}

Table 6 Cost of material provided by supplier $s$ for scenario 1

\begin{tabular}{ccccc}
\hline \multirow{2}{*}{ Supplier $s$} & \multicolumn{4}{c}{ Raw material $r$} \\
\cline { 2 - 5 } & 1 & 2 & 3 & 4 \\
\hline 1 & 2 & 2 & 2 & 3 \\
2 & 2 & 2 & 2 & 3 \\
3 & 1 & 1 & 1 & 3 \\
4 & 1 & 1 & 2 & 3 \\
5 & 2 & 1 & 3 & 2 \\
\hline \multicolumn{4}{c}{ For scenarios 2 and 3, the estimations are } \\
\multicolumn{4}{c}{ multiplied by 1.1 and 1.2 respectively. }
\end{tabular}
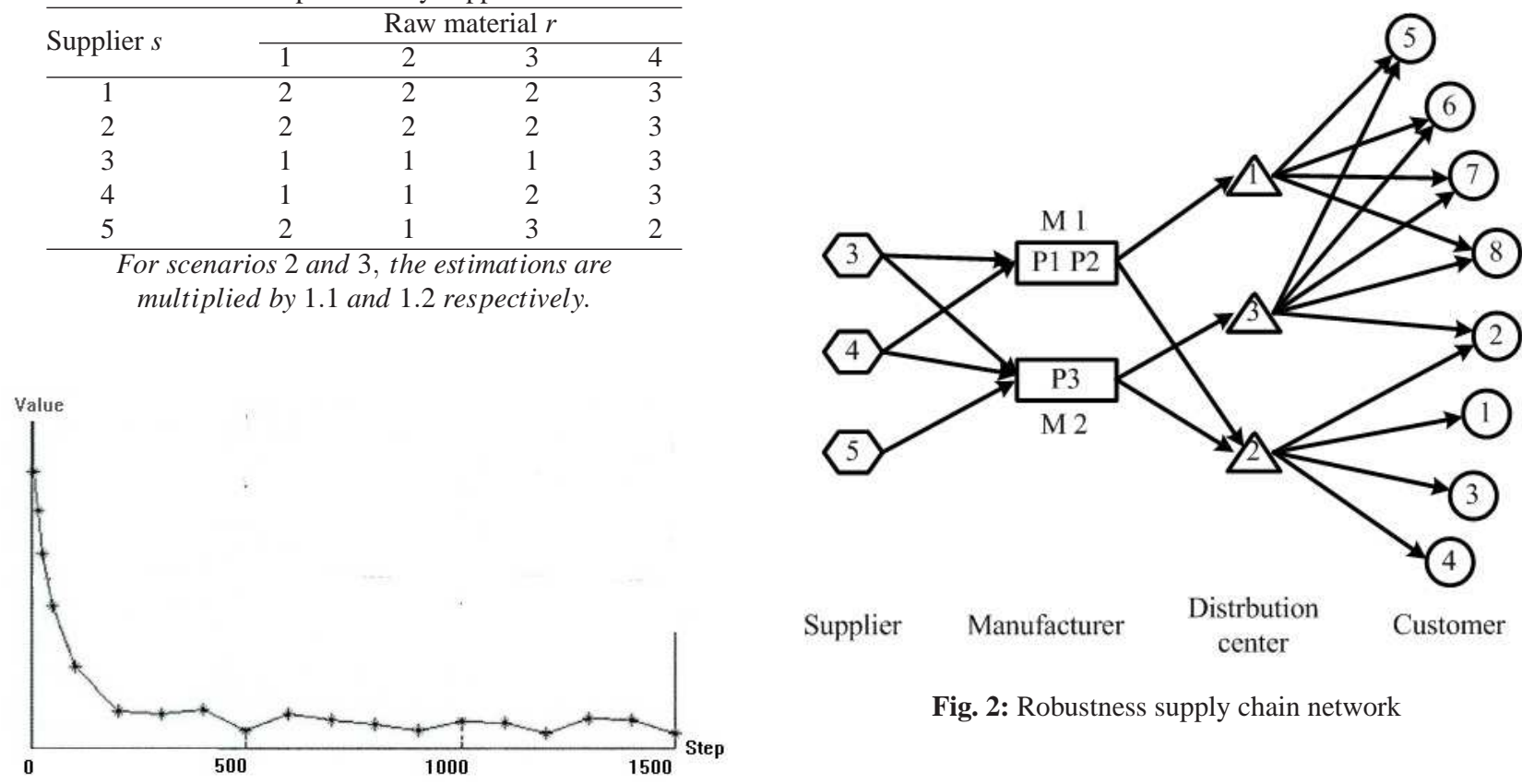

Fig. 2: Robustness supply chain network

Fig. 1: Convergence curves of the algorithm

By testing convergence time of different scale example, we have get that the convergence time varies from 30 to 450 second, so the computational time of the algorithm is also acceptable.

\subsection{Result}

\subsubsection{Robustness supply chain network}

As show in figure 2, the number in the rectangular frame represents the type of product produced by the manufacturer, for example, manufacturer 1 produce product 1 and product 2 , manufacturer 2 produce product 3 . The product in manufacturer

1 is transported to distribution center 1 and 2 , product 3 is transported to distribution center 2 and 3 . For satisfying the market demand, product 1 and 2 for customer 1, 2, 3, 4 are supplied by distribution center 2 , product 1 and 2 for customer $5,6,7,8$ are supplied by distribution center 1 , product 3 are supplied by distribution center 3 .

\subsubsection{The error analysis}

In this section, we compare the robust optimization model with stochastic programming model from the error of the model, since stochastic programming model is used average value to represent the objective function, there is no higher order term to punish the deviation of scenarios. So the objective function of stochastic programming model is: 
Table 5 Transportation cost to customer's zone $c$ for scenario 1

\begin{tabular}{ccccccccc}
\hline \multirow{2}{*}{$\begin{array}{c}\text { Distribution } \\
\text { center } l\end{array}$} & 1 & 2 & 3 & 4 & 5 & 6 & 7 & 8 \\
\hline 1 & 0.06 & 0.096 & 0.12 & 0.108 & 0.02 & 0.064 & 0.064 & 0.072 \\
2 & 0.03 & 0.06 & 0.04 & 0.05 & 0.04 & 0.072 & 0.08 & 0.096 \\
3 & 0.036 & 0.048 & 0.05 & 0.06 & 0.03 & 0.05 & 0.07 & 0.04 \\
4 & 0.03 & 0.036 & 0.03 & 0.05 & 0.08 & 0.072 & 0.024 & 0.064 \\
5 & 0.028 & 0.014 & 0.03 & 0.05 & 0.096 & 0.08 & 0.06 & 0.072 \\
\hline
\end{tabular}

Table 7 Cost of production and processing for scenario 1

\begin{tabular}{ccc|ccccc}
\hline \multirow{2}{*}{ Product $p$} & \multicolumn{3}{c}{ manufacturer $m$} & \multicolumn{5}{c}{ distribution center $i$} \\
\cline { 2 - 7 } & 1 & 2 & 1 & 2 & 3 & 4 & 5 \\
\hline 1 & 4 & 4.5 & 1.3 & 1.2 & 1.5 & 1.3 & 1.4 \\
2 & 5 & 5 & 1.3 & 1.2 & 1.4 & 1.2 & 1.5 \\
3 & 6 & 5.5 & 1.4 & 1.2 & 1.3 & 1.4 & 1.5 \\
\hline \multicolumn{2}{r}{ For scenarios 2 and 3, the estimations are multiplied by 1.1 and 1.2 respectively. }
\end{tabular}

Table 8 Cost of capacity purchase for scenario 1

\begin{tabular}{|c|c|c|c|c|c|c|c|}
\hline \multirow{2}{*}{ scenario $\xi$} & \multicolumn{2}{|c|}{ manufacturer $m$} & \multicolumn{5}{|c|}{ distribution center $i$} \\
\hline & 1 & 2 & 1 & 2 & 3 & 4 & 5 \\
\hline 1 & 1 & 1.2 & 0.6 & 0.7 & 0.6 & 0.8 & 0.7 \\
\hline 2 & 1.1 & 1.32 & 0.66 & 0.77 & 0.66 & 0.88 & 0.77 \\
\hline 3 & 1.2 & 1.44 & 0.72 & 0.84 & 0.72 & 0.96 & 0.84 \\
\hline
\end{tabular}

$$
\operatorname{Minf}=\sum_{\xi} \rho_{\xi} f^{\xi}
$$

Assumed the error between the supply and the actual product demand is:

$$
E_{j p}^{\xi}=\frac{\bar{q}_{p j}^{\xi}-D_{p j}^{\xi}}{D_{p j}^{\xi}} \times 100 \%
$$

So the error of the model is:

$$
E^{\xi}=\sum_{j} \sum_{p} E_{j p}^{\xi}
$$

The standard deviation of the error is:

$$
\sigma=\sqrt{\sum_{j} \sum_{p} \sum_{\xi}\left(E_{j p}^{\xi}\right)^{2} J P \xi}, J=8, P=3, \xi=9 \text {. }
$$

$E_{R}^{\xi}, \sigma_{R}$ denote the error and standard deviation of the robust optimization model respectively, $E_{S}^{\xi}, \sigma_{S}$ denote the error and standard deviation of the stochastic programming model respectively. The result shows in the table .

From table 9 we can see that, the error of robust optimization model varies from $0.51 \%$ to $9.38 \%$, and the standard deviation is $14.95 \%$. The error of stochastic programming model varies from $4.26 \%$ to $12.57 \%$, standard deviation is $25.61 \%$. The data shows that, through the establishment of robust optimization model of supply chain network, effectively improve the supply chain's ability to overcome the perturbation of the designed parameter, the ability of meeting uncertain market demand is enhanced.

\subsubsection{Risk coefficient analysis}

In order to consider the distribution of the objective function and make the model be suitable for high risky decision making under uncertainty, $\lambda \sum_{\xi} \rho_{\xi}\left(f^{\xi}-\sum_{\xi} \rho_{\xi} f^{\xi}\right)^{2}$ is added.

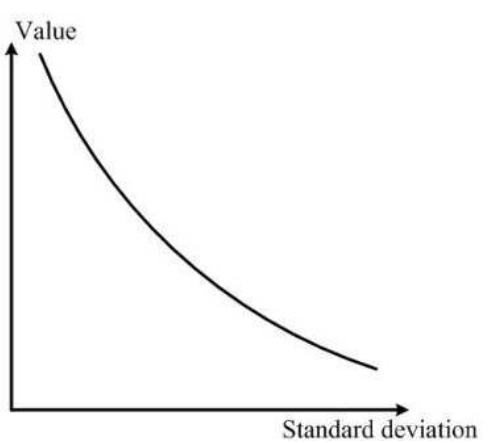

Fig. 3: Boundary curve of risk coefficient

We fix the weight of the objective programming, set $\omega=$ 5 , structure the boundary curve which shows objective function value and standard deviation change with $\lambda$. As show in figure 3 , with the increase of $\lambda$, deviation decreases, but the value of objective function increases. However, if $\lambda$ decreases, the value of objective function decreases, but the deviation increases.

\section{Conclusion}

This paper addresses a research on supply chain network design, under the condition of uncertain market demand and cost, we 


\begin{tabular}{cccccccccc}
\multicolumn{8}{c}{ Table 9 Error of meeting the market demand } \\
\hline$\xi$ & 1 & 2 & 3 & 4 & 5 & 6 & 7 & 8 & 9 \\
\hline$E_{R}^{\xi}$ & $4.28 \%$ & $3.56 \%$ & $9.38 \%$ & $0.51 \%$ & $0.85 \%$ & $2.34 \%$ & $5.32 \%$ & $6.48 \%$ & $5.28 \%$ \\
$E_{S}^{\xi}$ & $7.74 \%$ & $8.29 \%$ & $12.57 \%$ & $5.29 \%$ & $4.26 \%$ & $6.78 \%$ & $9.36 \%$ & $10.49 \%$ & $8.92 \%$ \\
\hline \multicolumn{8}{c}{$\sigma_{R}=8.685 \%, \sigma_{s}=15.367 \%}$.
\end{tabular}

proposed a effective method to make facility location decisions as well as capacity allocation, facilities allocation and the choice of supplier. The supply chain network can not only reduce the sensitivity of parameter perturbation, but also can effectively control the cost.

The decomposition and coordination strategy is adapted, first we build the selecting supplier sub-model and facility decision sub-model respectively, then integrate the two model reasonably and propose the robust optimization model under uncertainty condition. When solving the model, we decompose the model as two parties: the first part is facility location decision which only concludes $0-1$ decision variable, tabu search algorithm is used to determine the $0-1$ decision variables, and then regard the $0-1$ variables as known input parameters. The second part is flow decision, all-or-nothing method is proposed to design the capacity of the facility. From the numerical example, we can see that, the model and algorithm is valid and effective, the robust optimization model not only reduces the risk of market, but avoids the error from the shortage cost.

However, there are many shortage in this paper. In order to simply the model and convenient to solve, some factors are ignored, such as, the inventory holding cost, back-order cost, environmental cost and so on. Multi-objective function should be established to more accurately reflect the actual situation of the operation of the supply chain with real social effect.

\section{Acknowledgement}

This work was supported by Major International Joint Research Program of the National Natural Science Foundation of China (71020107027), National High Technology Research and Development Major Program of China (Program 863) (2008AA04A107), Central University Fund of Sichuan Unversity No. skqy201112.

\section{References}

[1] D. Simchi-Levi and P. Kaminsky, Irwin McGraw-Hill, Boston, (2004).

[2] C. C. Lin and T. H. Wang, Transportation Research: Part B, 45, (2011).

[3] Walid Klibi, Alain Martel and Adel Guitouni, European Journal of Operational Research, 203, (2010).

[4] A. M. Geoffrion and R. F. Powers, Interfaces, 25, (1995).

[5] M. Goetschalckx, C. J. Vidal and K. Dogan, European Journal of Operational Research, 143, (2002).

[6] H. Yan, Z. Yu and T. C. E. Cheng, Computers and Operations Research, 30, (2003).
[7] A. Amiri, European Journal of Operational Research, (2004).

[8] J. M. Mulvey, R. J. Vanderbei and S. A. Zenios, Operations Research, 43, (1995).

[9] C. S. Yu and H. L. Li, International Journal of Production Economics, 64, (2000).

[10] T. Santoso, S. Ahmed , M. Goetschalckx, and A. Shapiro, European Journal of Operational Research, 167, (2005).

[11] A. Azaron, K. N. Brown, S. A. Tarima and M. Modarres, International Journal of Production Economics, 116, (2008).

[12] P. Schütz, A. Tomasgard, and S. Ahmed, European Journal of Operational Research, 199, (2009).

[13] P. Feng and N. Rakesh, Computers and Operations Research, 37, (2010).

[14] A. BenTal, B. D. Chung, S. R. Mandala and T. Yao, Computers and Industrial Engineering, 45, (2011).

[15] M. S. Pishvaee, M. Rabbani and S. A. Torabi, Applied Mathematical Modelling, 35, (2011).

[16] G. J. Hahn and H. Kuhn, International Journal of Production Economics, 139, (2012).

[17] Atefeh Baghalian, Shabnam Rezapour, Reza Zanjirani Farahani, European Journal of Operational Research, 227, (2013). 


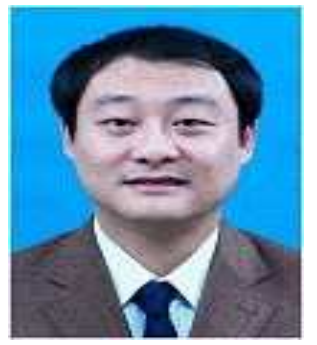

Maozhu Jin is an instructor of Business School,the tutor of MBA operations management and innovation and entrepreneurship management in Sichuan University. He has been engaged in the teaching of core curriculums such as operations management and management consulting. His current research interests include the areas of operations management, organizational process reengineering, strategic management, service operations management, platform-based mass customization and risk management. He has published two books and over ten research papers in authoritative journals of high quality both at home and abroad, and ten of them are retrieved by SCI and EI.

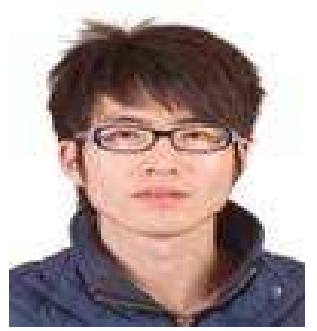

Ruimin Ma is a doctoral student in Sichuan University for majoring in management science and engineering, and a member of Information and Business Management Institute of Sichuan University. His researches mainly relate to simulation, multi-objective decision, and vehicle scheduling, etc.

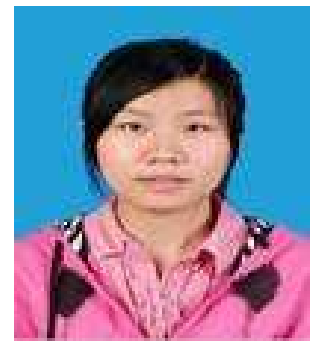

Lifei Yao is a Graduate student in Sichuan University for majoring in management science and engineering, and a member of Information and Business Management Institute of Sichuan University. Her research interests are prediction, evaluation, decision control and vehicle scheduling.

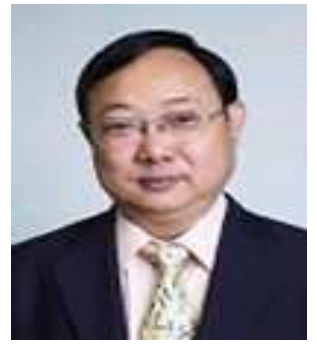

Peiyu Ren is a Professor, $\mathrm{PhD}$ Supervisor, currently acting as the Director of Information and Business Management Research Institute of Sichuan University. $\mathrm{He}$ has presided over and completed five surface projects of National Natural Science Foundation of China, being in charge of project research of Projects 863, 985 and 211, having published 15 books, monographs and more than 100 academic papers, including SCI, EI and CSSCI. 